\title{
Spontaneous and reflex activity of facial muscles in dystonia, Parkinson's disease, and in normal subjects
}

Günther Deuschl, Christof Goddemeier

\begin{abstract}
Objective-The blink rate is an index which can be easily obtained during the clinical examination, but it has not yet been properly standardised. The present study was undertaken to collect data on the age dependent development of this index and on possible abnormalities in Parkinson's disease and dystonia.

Methods-The blink rate and the rate of perioral movements were measured in 156 normal controls, 51 patients with Parkinson's disease, 48 patients with spasmodic torticollis, 14 patients with generalised dystonia, and 12 patients with focal hand or leg dystonias and have been correlated with the results of testing the orbicularis oculi reflex, the palmomental reflex, and the perioral reflex.
\end{abstract}

Results-No age related effects were found for the blink rate and perioral movements but all the reflexes showed age dependent variations. It is sufficient to measure the blink rate for one minute, provided standardised conditions are applied. Blink rate and perioral movement rate were positively correlated in patients and controls. The blink rate was significantly increased in spasmodic torticollis and decreased in Parkinson's disease. In generalised dystonia the blink rate was increased but in hand and leg dystonia the blink rate was normal. The reflex tests did not significantly differ between the subject groups except for the orbicularis oculi reflex, which was hyperexitable in Parkinson's disease.

Conclusion-Measuring the blink rate can assist the diagnosis of extrapyramidal disorders as a soft sign, but is not very sensitive. The group differences found indicate a decrease of the blink rate and perioral movements in hypokinetic and an increase in hyperkinetic extrapyramidal disorders such as spasmodic torticollis and generalised dystonias. This may be of interest for future pathophysiological studies.

(F Neurol Neurosurg Psychiatry 1998;64:320-324)

Keywords: blink rate; orbicularis oculi reflex; primitive reflexes; spasmodic torticollis; Parkinson's disease

Blinking protects the conjunctiva from drying and other possible injuries. Several external conditions such as humidity and smoking fume content increase the blink rate. ${ }^{12}$ Complete darkness or blindness change blink rate only to a limited extent. ${ }^{1}$ However, physiological factors related to the subjects' general status including attention seem to be much more important. $^{12}$

From the neurological point of view central regulation of the blink rate is of special interest as different pathological conditions can modulate the blink rate. Reduction of the blink rate has been found in Parkinson's disease, ${ }^{3}$ in progressive supranuclear palsy, ${ }^{4}$ and in subjects taking dopamine receptor blockers. ${ }^{5}$ This reduction is considered to be such a well defined feature of Parkinson's disease that it is even part of an item of the unified Parkinson's disease rating scale. ${ }^{6}$ An increase of the blink rate has not yet been established as a diagnostic sign although it has been found in some patients with Huntington's disease ${ }^{7}$ or blepharospasm. $^{2}{ }^{7-10}$

The anatomical pathways and physiological connections involved in the central control of blinking have been reviewed recently. ${ }^{11}$ The parapontine reticular formation and the lateral geniculum are known to facilitate eye blinking. ${ }^{212}$ The cerebellum seems to inhibit blinking ${ }^{2}$ because the removal of the cerebellum increases blink rate in rats. ${ }^{7}$ The basal ganglia play an important part in regulating the frequency of the eye blinks ${ }^{13}$ but further and more complex aspects of levator palpebrae and orbicularis oculi interaction such as their mutual reciprocal inhibition and especially the levator palpebrae inhibition ${ }^{814}$ are under control of basal ganglia circuits.

Little is known about age related changes in blink rate. The blink rate of newborn humans is below the values of adults ${ }^{15}$ and slowly increases until the age of 20 years. The blink rate seems to be modulated significantly by the testing paradigms: It is lower during reading than during a relaxed conversation. ${ }^{16}$ The available data suggest that a protocol for blink reflex measurements has to be standardised first before more extended measurements in patients seem to be valuable. Thus we selected our testing paradigms in a methodological pilot study before the normative study was started. The normative study assessed the blink rate in a large group of normal subjects with special emphasis on elderly people. Finally, blink rate was measured in a group of patients with hypokinetic (Parkinson's disease) and a group with hyperkinetic (focal and generalised dystonia) movement disorders. Some additional clinical indices such as the number of perioral movements and some facial reflexes 


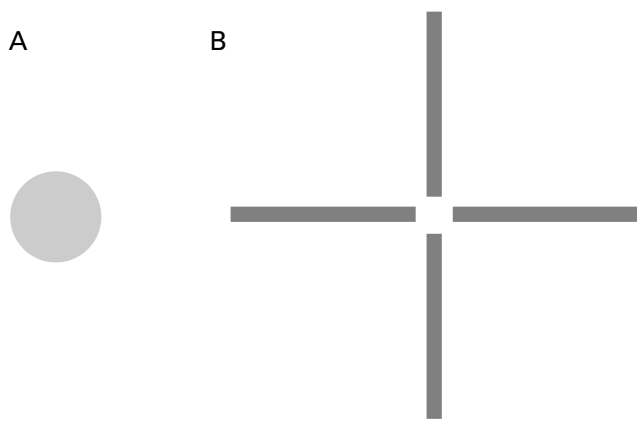

Figure 1 Symbols used to fixate used in the present study displayed at a distance of about $50 \mathrm{~cm}$. (A) point with an original size of $2 \mathrm{~cm}$. (B) open cross with an edge size of $9.5 \mathrm{~cm}$.

(palmomental reflex, orbicularis-oculi reflex, and perioral reflexes) were included because they are known to be related to normal aging or damage of central nervous functions. ${ }^{17}$

\section{Patients and methods}

BLINK RATE

A blink was defined as any visible, bilateral contraction of the orbicularis muscles-no matter if the eyes were completely closed. Two blinks were considered as separate, if they could be separated visually from each other. Blink rate was expressed as blinks/minute.

PERIORAL MOVEMENTS

Any visible perioral movement (mainly involving the lips) was rated as a perioral movement and two were considered separate if there was a pause between both. Perioral movements were rated as perioral movements/minute.

ORBICULARIS OCULI REFLEX

The definitions of Jensen et $a l^{17}$ were used. Briefly, adaptation was considered present (reflex negative) if the blinking was reduced or absent after 15 taps to the glabella. Any other response was considered as lacking adaptation (reflex positive). It was considered grade 1 if the adaptation was lacking only for the glabellar portion; grade 2 if adaptation was also lacking for the skin over the eyebrows; and grade 3 if the adaptation was lacking over the eyebrows until the hair boundaries.

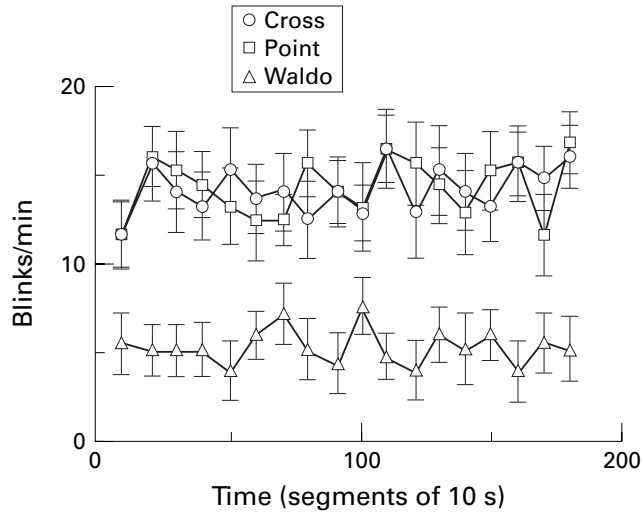

Figure 2 Blink rate measured over intervals between $60 s$ and $180 \mathrm{~s}$ in five normal subjects when fixating a searching task (Waldo), the point (task I), or the cross (task II) (displayed in fig 1). The blink rate is higher when fixating cross or point compared with the searching task. No fatigue effects were found during the observation period.

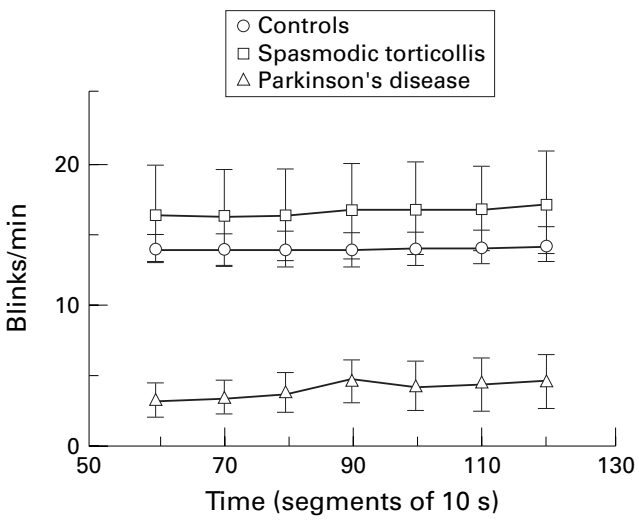

Figure 3 Blink rate measured over intervals of between 60 and 120 s in five normal subjects, five patients with Parkinson's disease, and five patients with spasmodic torticollis. The blink rate was already stable after 60 seconds of measurement.

PALMOMENTAL REFLEX

The palmomental reflex was considered positive when a sudden twinge to the thenar eminence caused a contraction of the ipsilateral $M$ triangularis.

PERIORAL REFLEXES

Perioral reflexes were considered to be positive if a sudden finger tap to the upper lip caused a visible contraction of the orbicularis oris muscle.

TESTING CONDITIONS

After a pilot phase (see below) the blink rate and the perioral movements were always measured under the following conditions: The blinks and perioral movements were measured in a relaxed atmosphere either in a quiet place in the hospital or in the subject's home. The subjects had to stare at an open cross with 9.5 $\mathrm{cm} \times 9.5 \mathrm{~cm}$ side length for two minutes (fig 1 ). The subjects were told that their facial expression would be noted. The light was not standardised. Blink rate and perioral movements were measured during this time. Subsequently the reflexes were tested. All the assessments were made by one of us (CG).

METHODOLOGICAL STANDARDISATION

In an attempt to standardise the measurement of blink rate and perioral movements for clinical bedside testing, three conditions were

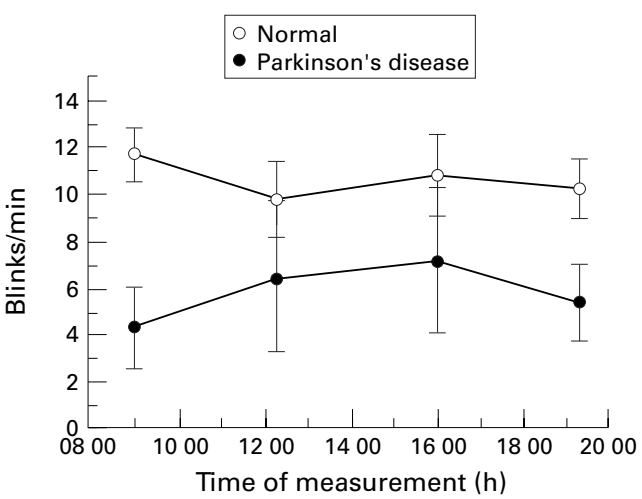

Figure 4 Blink rate measured three times a day in five patients with Parkinson's disease and five normal subjects. No consistent daytime variations were found. 

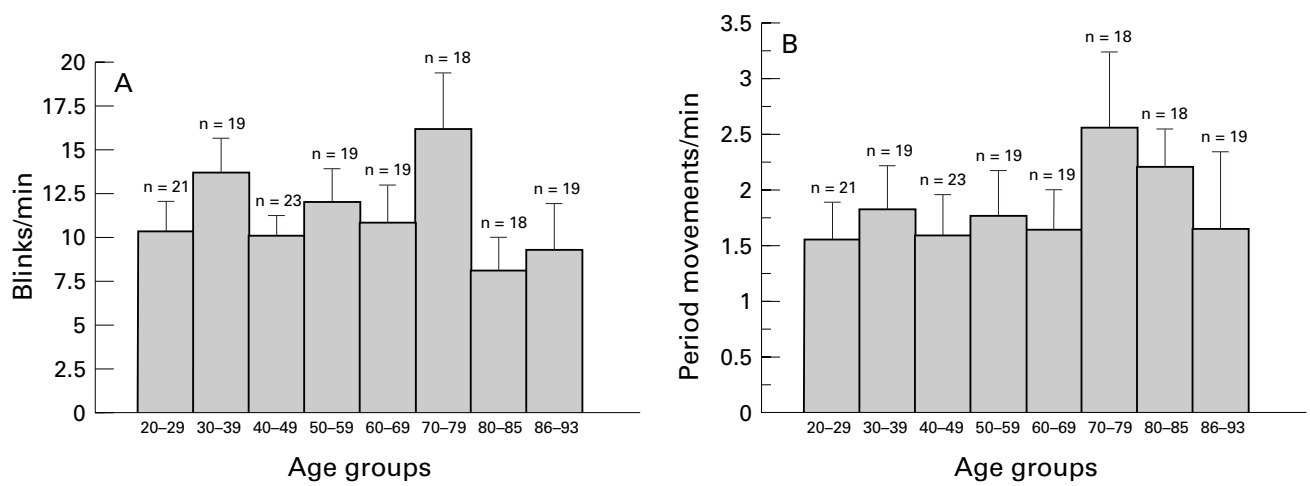

Figure 5 Blink rate $(A)$ and rate of perioral movements $(B)$ in 156 normal subjects displayed in age classes of 10 or 5 years respectively. Significant age related changes do not occur.

evaluated in a pilot study in five normal subjects, five patients with Parkinson's disease, and five patients with spasmodic torticollis. In a visual "screening task" the aim was to search for a target (the person "Waldo") in a commercial picture book. ${ }^{18}$ In "gaze fixation" the subjects were asked to stare at an open cross (task I) or a dot (task II) (fig 1). We avoided paradigms which were difficult to standardise (for example, measuring during relaxed conversation).

SUBJECTS

A total of 156 normal controls with special reference to the older age groups (mean age: 57.07, range: 21-93 years) was investigated.

They were compared with 51 patients with Parkinson's disease (stage 2-4, Hoehn and Yahr grade). The mean duration of their disease was 9 (SD 7.3) years. Forty nine of the 51 patients received medication including levodopa. The patients were all on medication.

The other patient groups were 48 patients with spasmodic torticollis (mean duration 8 (SD 5) years). They were mostly under treatment with botulinum toxin for their torticollis. The assessments were made at least four months after the last injection.

Twenty six patients with other dystonias were assessed. Fourteen had generalised dystonia including craniocervical segments (mean duration 11(SD 9) years). Eleven of these

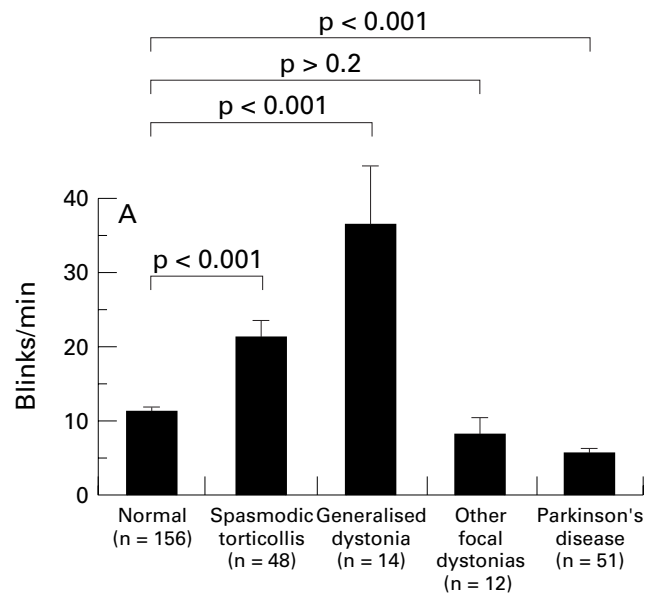

Figure 6 Blink rate $(A)$ and rate of perioral movements (B) in patients with Parkinson's disease, spasmodic torticollis, and generalised dystonia with craniocervical involvement and hand or leg dystonia compared with controls. Values are medians (SEM)

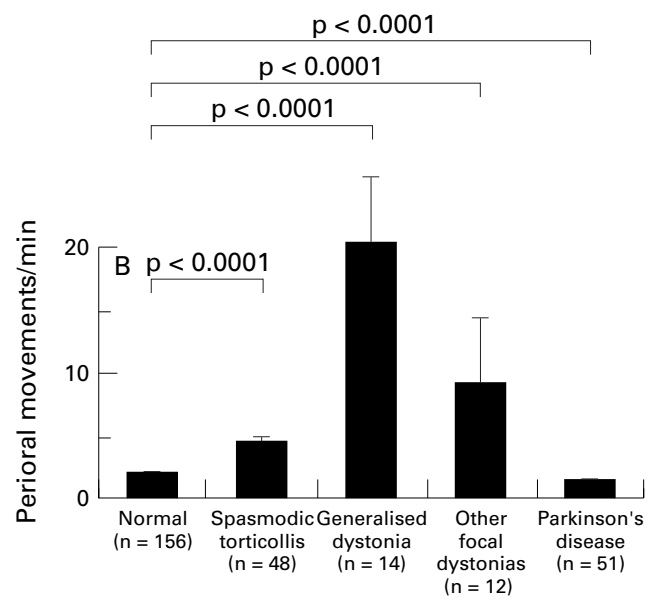

patients had idiopathic dystonia and three of them were suspected to have tardive dyskinesia. Twelve patients had focal dystonias of the hands or legs (mean duration 9.6 (SD 11) years) mostly due to focal brain lesions. Age and sex matched control groups were selected from the normal subjects for statistical comparisons.

\section{STATISTICS}

$\chi^{2}$ Tests, $U$ tests, regression analysis, and correlation analysis were applied. If the data were not normally distributed, they were transformed (mostly $\log (1+\mathrm{x})$ ). The results are displayed as mean (SEM).

\section{Results}

STANDARD MEASUREMENT

In all the subjects the screening task ("Waldo") was associated with a significantly lower blink rate than the visual fixation task ("dot" or "cross"). No significant difference was found between the fixation conditions (fig 2). As most of the subjects preferred to look at the open cross, this was chosen for the subsequent measurements.

To assess the time necessary to reliably measure the blink rate and perioral movements, five normal subjects and 10 patients were evaluated at time intervals between $60 \mathrm{~s}$ and $120 \mathrm{~s}$ (fig 3). The mean value for blink rate and also perioral movements (the second is not 
Table 1 Blink rate (BR) and perioral movements (POM) in controls and patients

\begin{tabular}{lcc}
\hline & BR Mean (SD) & POM Mean (SD) \\
\hline Controls & $11.3(9.1)$ & $1.9(1.8)$ \\
Parkinson's disease & $5.8(5.3)$ & $1.4(1.7)$ \\
Spasmodic torticollis & $21.3(16.2)$ & $4.3(4.4)$ \\
Generalised dystonia with craniocervical dystonia & $36.9(27.7)$ & $20.2(20.4)$ \\
Hand or leg dystonia & $7.4(8.2)$ & $9.0(18.8)$ \\
\hline
\end{tabular}

Table 2 Orbicularis oculi reflexes in controls and patients

\begin{tabular}{lcccc}
\hline Grade & $\begin{array}{l}\text { Controls } \\
\text { Abs }{ }^{*}(\%)\end{array}$ & $\begin{array}{l}\text { Parkinson's disease } \\
\text { Abs (\%) }\end{array}$ & $\begin{array}{l}\text { Spasmodic torticollis } \\
\text { Abs (\%) }\end{array}$ & $\begin{array}{l}\text { Other dystonia } \\
\text { Abs (\%) }\end{array}$ \\
\hline 0 & $65(42)$ & $2(4)$ & $17(35)$ & $10(38)$ \\
1 & $47(30)$ & $10(20)$ & $13(27)$ & $3(12)$ \\
2 & $35(22)$ & $24(47)$ & $16(33)$ & $9(35)$ \\
3 & $9(6)$ & $15(29)$ & $2(4)$ & $4(15)$ \\
Total & 156 & 51 & 48 & 26 \\
\hline
\end{tabular}

$\star$ Number of subjects.

shown in fig 3) were already stable after one minute and did not change significantly with longer observation periods. As expected, the largest variations occurred for the group with the lowest blink rate (Parkinson's disease). But these results suggest that measuring the blink rate for one minute is long enough to get stable values.

To study an eventual diurnal rhythm of blink rate or perioral movements, the blink rate and perioral movements were assessed in five normal subjects and five patients with Parkinson's disease four times a day (fig 4). We found no consistent diurnal variations.

BLINK RATE AND PERIORAL MOVEMENTS

Among the controls, neither the blink rate nor the perioral movements were clearly correlated with age or exhibited a tendency to higher or lower values in the older ages (fig 5). There were no sex related differences. The blink rate and the perioral movements were correlated in the normal subjects and the patient groups $(r=0.330, \mathrm{p}<0.0001)$.

Table 1 and figure 6 show the mean values of blink rate and perioral movements for all the subject groups. Due to the large scatter in the normal population the $95 \%$ confidence intervals are large. According to our normative data blink rates below $1 / \mathrm{min}$ and above $46 / \mathrm{min}$ are considered pathological. Ten per cent of the normal subjects had a blink rate below $2 / \mathrm{min}$ or above $23 / \mathrm{min}$. For perioral movements a lower border was not discernible but a frequency above $7 / \mathrm{min}$ was abnormal.

Table 3 Palmomental reflex (right or left) in controls and patients

\begin{tabular}{lllll}
\hline Grade & $\begin{array}{l}\text { Controls } \\
\text { Abs (\%) }\end{array}$ & $\begin{array}{l}\text { Parkinson's disease } \\
\text { Abs (\%) }\end{array}$ & $\begin{array}{l}\text { Spasmodic torticollis } \\
\text { Abs (\%) }\end{array}$ & $\begin{array}{l}\text { Other dystonia } \\
\text { Abs (\%) }\end{array}$ \\
\hline 0 & $124(79)$ & $29(57)$ & $36(75)$ & $23(88)$ \\
1 & $32(21)$ & $22(43)$ & $12(25)$ & $3(12)$ \\
Total & 156 & 51 & 48 & 26 \\
\hline
\end{tabular}

Table 4 Perioral reflexes in controls and patients

\begin{tabular}{lllll}
\hline Grade & $\begin{array}{l}\text { Controls } \\
\text { Abs (\%) }\end{array}$ & $\begin{array}{l}\text { Parkinson's disease } \\
\text { Abs (\%) }\end{array}$ & $\begin{array}{l}\text { Spasmodic torticollis } \\
\text { Abs (\%) }\end{array}$ & $\begin{array}{l}\text { Other dystonia } \\
\text { Abs (\%) }\end{array}$ \\
\hline 0 & $138(88)$ & $39(76)$ & $44(92)$ & $25(96)$ \\
1 & $18(12)$ & $12(24)$ & $4(8)$ & $1(4)$ \\
Total & 156 & 51 & 48 & 26 \\
\hline
\end{tabular}

Compared with normal subjects, both the blink rate and the perioral movements had significantly higher values in patients with spasmodic torticollis and significantly lower values in patients with Parkinson's disease (fig 6). In generalised dystonia, the blink rate and the perioral movements were significantly higher whereas it was normal for the hand and leg dystonias.

\section{REFLEXES}

Tables 2-4 show the results of reflex testing (orbicularis oculi reflex, palmomental reflex, and perioral reflexes). All the reflexes showed an increase of positive findings with increasing age $\left(\mathrm{p}<0.0001\right.$, logistic regression, $\chi^{2}$ test $)$. Thus any differences between normal subjects and patients had to be corrected for age. Orbicularis oculi reflexes were significantly $(p<0.0001)$ more often abnormal in Parkinson's disease but were normal in spasmodic torticollis or segmental/generalised dystonia. No differences were found between controls and patients for palmomental and perioral reflexes. Neither the blink rate nor the perioral movements were correlated with the increased incidence of orbicularis oculi reflexes in Parkinson's disease.

\section{Discussion}

The present study standardised blink rate and perioral movement testing in normal subjects and assessed patients with two basal ganglia diseases, a hyperkinetic and a hypokinetic one. Blink rate and perioral movements decreased in Parkinson's disease and increased in focal and generalised dystonia and were highly correlated with each other. The facial nerve reflexes tested showed a systematic age dependent effect but when corrected for age, only the blink reflex showed significant abnormalities in Parkinson's disease, but not in dystonia. The blink rate and perioral movements showed no sex related differences.

We confirmed and extended the view that the blink rate can be reliably measured by means of clinical observation only. The mean value of $11.38 \mathrm{blinks} / \mathrm{min}$ is similar to that found in previous smaller studies. ${ }^{1{ }^{15}}{ }^{19}{ }^{20}$ It has been shown that the blink rate differs at rest (19-20 blinks $/ \mathrm{min}^{1321}$ ), during conversation (25-29 blinks $\left./ \min ^{13}{ }^{16}{ }^{21}\right)$, and when reading (3.3-14 blinks $\left./ \mathrm{min}^{13}{ }^{16}{ }^{21}\right)$. As all these conditions cannot be standardised, we decided to provide always the same visual stimulus. The present findings show that this test is standardised and reproducible. The values obtained for perioral movements were correlated with the blink rate and the group comparisons (except for generalised dystonia) showed the same differences for both indices. Thus we consider it sufficient to assess the blink rate only in future studies.

We confirmed age related changes in facial reflexes with a clear cut age dependent increase in positive findings but were surprised to find that spontaneous facial movements differed in their development because blink rate and perioral movements were neither more nor less frequent at older ages. 
Patients with Parkinson's disease have a lower blink rate and less perioral movements than normal subjects. This is already well known ${ }^{314}$ but the blink rate of only 11 of 51 patients $(21 \%)$ with Parkinson's disease was below the $95 \%$ confidence interval of normal subjects and only 18 (35\%) had a blink rate below $2 \mathrm{~Hz}$. Most likely this is because our patients were all treated with levodopa and dopamine agonists and dopaminergic medication can increase the blink rate. ${ }^{1322} \mathrm{It}$ remains to be determined if lower values will be found in a drug naive population or in patients off medication for 12 hours. Interestingly the orbicularis oculi reflexes are often abnormal in Parkinson's disease but perioral reflexes and palmomental reflexes are not. This has been found in other studies ${ }^{172324}$ and is generally considered as a disease specific loss of inhibition of brainstem neurons.

This study shows for the first time that the blink rate and the perioral movements are both increased in spasmodic torticollis. Similar findings have hitherto only been made in some patients with blepharospasm. ${ }^{25}$ Moreover, a group with generalised dystonias has been analysed. Whenever they had cranial nerve involvement, the blink rate and perioral movements were even more increased as in torticollis. By contrast patients with arm or leg dystonias or hemidystonia had a normal blink rate. Thus a higher blink rate seems to be restricted to some forms of dystonias and might reflect a subtle sign of basal ganglia dysfunction when the dystonia involves the craniocervical region. It seems to be an attractive hypothesis that dystonia, as the most important variant of a hyperkinetic movement disorder, is associated with an increased blink rate. At present such a statement seems to be justified only for spasmodic torticollis, blepharospasm, and generalised dystonias with cranial nerve involvement and future studies are needed to extend these findings.

By contrast with our findings in Parkinson's disease we have no evidence that the clinical facial nerve reflex tests applied in the present study are abnormal in spasmodic torticollis or generalised dystonia. This is relevant with respect to the changes of inhibitory cranial nerve reflexes and other reflexes in various forms of focal and generalised dystonia which have been shown earlier with electrophysiological tests. ${ }^{26-30}$ In other words, the abnormalities of cranial nerve reflex circuits in dystonia do not show up in the clinical reflex tests of the present study.

In conclusion, blink rate and perioral movements are significantly abnormal in basal ganglia diseases affecting the head and neck. As a diagnostic test it is not very sensitive either for Parkinson's disease under dopaminergic treatment or for spasmodic torticollis or generalised dystonia. However, both may serve as extrapyramidal "soft signs" because they exhibit clear cut group differences to a normal control population. Moreover, these tests provide an easy tool to study differences of clinical indices with possible pathophysiological relevance in diseases which are probably related to basal ganglia dysfunction.

We thank all the subjects and patients for their active contribution, Dr G Fuchs, Parkinson-Klinik Wolfach, for allowing us to study patients under his care, and Professor J Schulte-Mönting, Institute for Medical Statistics, University of Freiburg, for help with the statistical analysis of the data.

1 Ponder E, Kennedy WP. On the act of blinking. Quarterly fournal of Experimental Physiology 1928;18:89-110

2 Karson CN. Physiology of normal and abnormal blinking. Adv Neurol 1988;49:25-37.

3 Karson CN, LeWitt PA, Calne DB, et al. Blink rates in parkinsonism. Ann Neurol 1982;12:580-3.

4 Pfaffenbach DD, Layton DD, Keans TD. Ocular manifestations in progressive supranuclear palsy. Am 7 Ophthalmol 1972;74:1179-84

5 Karson CN, Freed WJ, Kleinman JE, et al. Neuroleptics decrease blinking in schizophrenic subjects. Biol Psychiatry 1981;16:679-82

6 Goetz CG, Stebbins GT, Chmura TA, et al. Teaching tape for the motor section of the unified Parkinson's disease rating scale. Mov Disord 1995;10:263-6.

7 Karson CN, Burns RS, LeWitt PA, et al. Blink rates and disorders of movement. Neurology 1984;34:677-8.

8 Aramideh M, Bour LJ, Koelman JH, et al. Abnormal eye movements in blepharospasm and involuntary levator palpebrae inhibition. Clinical and pathophysiological considerations. Brain 1994;117:27-38.

9 Manning KA, Evinger C, Sibony PA. Eyelid movements before and after botulinum therapy in patients with lid spasm. Ann Neurol 1990;28:653-60.

10 Valls-Sole J, Tolosa ES, Ribera G. Neurophysiological observations on the effects of botulinum toxin treatment in patients with dystonic blepharospasm. 7 Neurol Neurosurg Psychiatry 1991;54:310-13.

11 Schmidtke K, Büttner-Ennever JA. Nervous control of eyelid function: a review of clinical, experimental and pathological data. Brain 1992;115:227-47.

12 Cohen B, Feldman M. Relationship of electrical activity in pontine reticular formation and lateral geniculate body to pontine reticular formation and lateral geniculate body

13 Karson CN. Spontaneous eye-blink rates and dopaminergic ystems. Brain 1983;106:643-53.

14 Löffler JD, Slatt B, Hoyt WF. Motor abnormalities of the eyelids in Parkinson's disease. Arch Ophthalmol 1966;76: $178-85$

15 Zametkin AJ, Stevens JR, Pittman R. Ontogeny of spontaneous blinking and of habituation of the blink reflex. Ann Neurol 1979;5:453-57.

16 Hall A. The origin and purposes of blinking. $\mathrm{Br} F$ Ophthalmol 1945;29:445-67.

17 Jensen JPA, Gron U, Pakkenberg $\mathrm{H}$. Comparison of three primitive reflexes in neurogical patients and in normal
individuals. $\mathcal{F}$ Neurol Neurosurg Psychiatry 1983;46:162-7.

18 Handford M. The great Waldo search. Boston: Little Brown, 1989.

19 Carney LG, Hill RM. The nature of normal blinking patterns. Acta Ophthalmol Scand 1982;60:427-33.

20 Strempel I. Zum Problem des "Trockenen Auges". Deutsches Ärzteblatt 1994;204-8.

21 Bentivoglio AR, Bressman SB, Cassetta E, et al. Analysis of blink rate in a cohort of healthy volunteers. Mov Disord 1994;9:49.

22 Karson CN, Staub RA, Kleinman JE, et al. Drug effect on blink rates in rhesus monkeys: prelimimary studies. Biol Psychiatry 1981;16:249-54

23 Pearce J, Aziz H, Gallagher JC. Primitive reflex activity in primary and symptomatic parkinsonism. F Neurol Neurosurg Psychiatry 1968;31:501-8.

24 Klawans HL, Goodwin JA. Reversal of the glabellar reflex in parkinsonism by L-dopa. I Neurol Neurosurg Psychiatry 1969;32:423-7.

25 Jankovic J, Orman J. Blepharospasm: demographic and clinical survey of 250 patients. Ann Ophthalmol 1984;16: 371-6.

26 Berardelli A, Rothwell JC, Day BL, et al. Pathophysiology of blepharospasm and oromandibular dystonia. Brain 1985; 50:593-608.

27 Pauletti G, Berardelli A, Cruccu G, et al. Blink reflex and the masseter inhibitory reflex in patients with dystonia. Mov Disord 1993;8:495-500.

28 Topka H, Hallett M. Perioral reflexes in orofacial dyskinesia and spasmodic dysphonia. Muscle Nerve 1992;15:1016-22.

29 Nakashima K, Rothwell JC, Thompson PD, et al. The blink reflex in patients with idiopathic torsion dystonia. Arch Neurol 1990;47:413-6.

30 Deuschl G, Seifert C, Heinen F, et al. Reciprocal inhibition of forearm flexor muscles in spasmodic torticollis. $\mathcal{F}$ Neurol Sci 1992;113:85-90. 